\title{
Análise da confiabilidade humana em um laboratório de análises clínicas utilizando FMEA e FTA
}

\section{Analysis of human reliability in a clinical laboratory using FMEA and Fault Trees}

Ariane Ferreira Porto Rosa ${ }^{1 *}$, Jordana Rubin Brito ${ }^{1}$, Rogério Royer ${ }^{1}$, Gilberto Tavares dos Santos $^{2}$

\section{RESUMO}

A análise de confiabilidade humana surge como suporte para quantificar e qualificar as falhas relacionadas às pessoas na execução de processos. Este trabalho apresenta um estudo de caso com 0 objetivo analisar a confiabilidade humana nos processos de atendimento de clientes e coleta de material biológico em um laboratório de análises clínicas. A pesquisa constituiu-se das seguintes etapas: revisão da literatura sobre os conceitos de falhas humanas e métodos para análise dessas falhas; mapeamento dos processos a fim de caracterizar falhas, levantamento do quantitativo de falhas por tipo e processo; análise do risco das falhas por meio da aplicação do método FMEA (Failure Mode and Effect Analysis); análise das causas das falhas, com o suporte de FTA (Fault Tree Analysis); e conclusões acerca das análises realizadas durante a pesquisa. Os levantamentos e análises realizados permitiram categorizar as falhas entre erros e transgressões e priorizar ações para preveni-las. Constatou-se que a ocorrência dessas falhas pode ser reduzida, se a qualificação profissional dos funcionários for consolidada.

Palavras-chave: Confiabilidade Humana; Falhas; Erros; Transgressões; FMEA; FTA.

\begin{abstract}
Human reliability analysis emerges as a support for quantifying and qualifying people-related failures in process execution. This work aims to analyze the human reliability in customer service processes and collection biological material in a laboratory of clinical analysis. The research consisted of the following steps: literature review about human failures and methods to analyze them; mapping of processes in order to characterize failures; quantification of failures by type and process; failure risk by using Failure Mode and Effect Analysis (FMEA); identification of failures causes by using Fault Tree Analysis (FTA); and conclusions about the analyzes carried out during the research. The surveys and analyzes made it possible to categorize failures between errors and transgressions and prioritize actions to prevent them. It was found that the occurrence of these failures can be reduced, if the professional qualification of the employees is consolidated.
\end{abstract}

Keywords: Human Reliability; Failures; Errors; Transgressions; FMEA; FTA.

${ }^{1}$ UFPel - Universidade Federal de Pelotas. *E-mail: afprosa61@gmail.com

${ }^{2}$ UFRGS - Universidade Federal do Rio Grande do Sul 


\section{INTRODUÇÃO}

O fenômeno da globalização vem provocando grandes mudanças no mundo dos negócios (COUTINHO e GOMES, 2012). É possível perceber que o ambiente organizacional, cada vez mais competitivo, tem feito com que as empresas sejam impulsionadas na busca de processos mais eficientes e que sejam capazes de atender aos clientes de forma cada vez mais eficiente.

Majoritariamente, esses processos são realizados por pessoas com o auxílio de tecnologia, sujeitando-se a falhas de duas origens, as mecânicas e as humanas. As falhas humanas são definidas por Lafraia (2001) como erros de ações planejadas em alcançar os objetivos propostos, que podem ocorrer na execução de uma atividade, e devem ser identificadas, minimizadas e quando possível evitadas.

Assim, a análise da confiabilidade humana (Human Analysis Reliability, HRA) surge como um estudo no qual as organizações devem recorrer para garantir a confiabilidade dos seus processos envolvendo pessoas. Pallerosi et al. (2011) definem confiabilidade humana como a probabilidade de que uma pessoa não falhe no cumprimento de uma tarefa, quando solicitada, em um determinado período de tempo. Para o autor, existem vários métodos para identificar, dimensionar, analisar e interpretar falhas humanas. Nesse sentido, muito dos métodos utilizados no tratamento de falhas mecânicas são adaptados para avaliar falhas humanas.

Entre as ferramentas para a identificação sistemática de falhas e riscos associados, destaca-se a Análise de Modos e Efeitos de Falhas (Failure Mode and Effect Analysis, FMEA), cujo conceito principal consiste em estabelecer uma sequência lógica e organizada de avaliação das possibilidades pelas quais um sistema ou processo está sujeito a falhar. A FMEA avalia a severidade das falhas, a forma como as mesmas podem ocorrer e como poderiam ser detectadas antes de chegarem ao cliente (FERNANDES, 2005).

Outra ferramenta que possibilita aprofundar os estudos de confiabilidade é Análise de Árvore de Falhas (Fault Tree Analysis, FTA). Trata-se de um método para avaliar as causas de falhas de forma estruturada e coerente (FOGLIATTO e RIBEIRO, 2009).

Desse modo, este trabalho visa realizar uma análise das falhas humanas que ocorrem nos processos de atendimento a clientes e coleta de material biológico em um laboratório de análises clínicas na cidade de Pelotas, RS, utilizando a FMEA e a FTA. 
O estudo de caso detalha-se nas seguintes etapas a serem abordadas na sequência: (i) apresentação de referencial teórico, (ii) definição de procedimentos metodológicos, e (iii) discussão dos resultados obtidos.

Assim, a problemática de pesquisa deste trabalho é: Como coletar, analisar e propor soluções para as falhas humanas em um processo de atendimento ao cliente e coleta de amterial biolégico em um laboratório de análises clínicas?

Embora o método de pesquisa proposto neste trabalho possa ser aplicado em outros casos similares, o estudo de caso realizado é único com foco em um produto e, portanto, os resultados obtidos delimitam-se ao escopo deste trabalho específico e que fortalece o método e suas ferramentas quando aplicado no geral, de modo a validar ganhos quando seguidos suas etapas precisamente.

O artigo está estruturado iniciando-se com a presente seção Introdução que delimita o escopo do trabalho desenvolvido. A seção 2 intitulada Referencial Teórico apresentaos conceitos dos principais assuntos teóricos como mapeamento de processos, falhas humanas, confiabilidade, FMEA e FTA. Na seção 3 Metodologia é apresentada a classificação da pesquisa e a proposta metodológica. A seção 4 trata do estudo de caso apresentando suas etapas e aplicação da metodologia proposta. Na seção 5 são apresentados os resultados obtidos no estudo de caso. A seção 6 apresenta as contribuições do trabalho para a indústria e sociedade. A seção 7 conclusões e sugestões de trabalhos futuros.

\section{REFERENCIAL TEÓRICO}

Nesta seção, contextualiza-se o referencial teórico em cinco tópicos que consubstanciam o desenvolvimento deste trabalho, quais sejam: (i) mapeamento de processos, (ii) falhas humanas e (iii) confiabilidade e HRA, (iv) FMEA, e (v) FTA.

\subsection{Mapeamento de processos}

Processo é um significado de extrema importância para representar os caminhos pelos quais uma empresa deseja produzir e entregar seus produtos e serviços aos clientes (ALVARENGA et al., 2013). De acordo com a NBR ISO 9000, um processo pode ser definido como um conjunto de recursos, atividades e pessoas inter-relacionadas que transformam insumos (entradas) em produtos (saídas). Seguindo essa premissa, uma 
técnica utilizada para otimização dos processos é o mapeamento de processos (ALVARENGA et al., 2013).

Para Slack et al. (2015), o mapeamento de processos é uma ferramenta que busca compreender e descrever os processos por meio de como as atividades se relacionam entre

si. É possível utilizar várias técnicas que buscam identificar o fluxo de materiais, pessoas ou informações que fazem parte de um processo. Trata-se de uma importante ferramenta visual, que busca demonstrar relações de dependência para a realização dos processos de trabalho (ALVARENGA et al., 2013).

Carpinetti (2016) afirma que o mapeamento de processos é uma representação gráfica e lógica do funcionamento de uma organização. O mapa, segundo o autor, tem o objetivo de mostrar pictoricamente o relacionamento entre seus elementos, estabelecendo-se por diagramas em que fluxos de atuação sejam compreensíveis para todos que se disponham a compreeender o andamento do processo. Silva et al. (2013) destaca como consequências de um bom mapeamento de processos a padronização por meio de documentos e definição de rotinas que assegurem a melhoria de desempenho desses processos e a sua manutenção.

\subsection{Falhas Humanas}

De acordo com o princípio básico apontado por Pallerosi et al. (2011) de que não existem pessoas a prova de falhas, deduz-se que os trabalhadores estão sujeitos a cometerem imperfeições na realização de uma tarefa. A expressão "falha humana no trabalho" significa que ocorreu um erro no funcionamento de um sistema e que foi causada ou teve contribuição de seres humanos (SILVA, 2006). Por consequência, a falha é a impossibilidade de um sistema ou componente cumprir sua função no nível especificado ou requerido (LAFRAIA, 2001).

O erro humano descreve as falhas psicológicas que podem causar a ação humana que leva à falha dos equipamentos. Devem ser levadas em consideração de forma diferente das falhas dos equipamentos que irão se degradar ao decorrer do tempo. Com a seleção por aptidão, treinamento frequente e consideração da experiência pessoal, as falhas humanas podem ser reduzidas ao longo do tempo (PALLEROSI et al., 2011). Além disso, para o autor, essas condições fazem com que as falhas possam ser previstas, quantificadas e, por consequência, minimizadas. 
Os autores também afirmam que as ações e omissões que induzem às falhas são caracterizadas pela não execução da atividade requerida ou a sua não realização de forma correta. As falhas humanas que ocorrem durante a execução das atividades humanas podem se dividir em erros e transgressões.

Quando a oscilação natural do comportamento humano produz algum resultado fora dos limites esperados, ou quando a capacidade humana para acompanhar as mudanças ambientais for insuficiente, resulta em erro (IIDA, 1991). Os erros constituem a principal parcela dos erros humanos e isto ocorre, pois dependem fundamentalmente da capacidade para executar a missão, do estressamento, das condições ambientais e sociais, da motivação na execução da tarefa e outros fatores. Os erros ocorrem involuntariamente, de forma "não consciente" (PALLEROSI et al., 2011).

Já as transgressões ocorrem pelas falhas comportamentais e devem ser separadas dos erros, para análises quantitativas, pois dependem de fatores especiais e difíceis de serem claramente delineados. Isso ocorre porque refletem mais as mazelas humanas relacionadas às condições de trabalho do que diretamente à capacidade física e mental para a execução das tarefas. Os autores afirmam que transgredir, por exemplo, significa desobedecer conscientemente a execução de algum procedimento, em prol de resolvê-lo mais rapidamente, o que o conduz à infração e, por vezes, ao erro (PALLEROSI et al., 2011).

\subsection{Análise da Confiabilidade Humana}

A confiabilidade pode ser definida como a probabilidade de que um componente, equipamento ou sistema cumprirá sua função sem falhas, por um período de tempo, sob condições especificadas (Lafraia, 2001). Para Fogliatto e Ribero (2009), a confiabilidade está relacionada a diversas áreas da Engenharia de Produção, tais como análises de riscos e segurança, qualidade, otimização da manutenção, proteção ambiental e projeto de produtos.

No que se refere às pessoas, a análise da confiabilidade humana (Human Reliability Analysis, HRA) manifesta-se como um estudo que se propõe a quantificar e qualificar as falhas que ocorrem nas operações e processos desempenhados pelas pessoas. Um princípio básico, proposto por Pallerosi et al. (2011), descreve que não existem pessoas à prova de falhas, ou seja, todos podemos falhar no desempenho de uma tarefa. Em complemento a isso, o conceito de ato inseguro vem sendo utilizado para projetar 
sobre o empregado a responsabilidade sobre acidentes causados por distorções na organização do trabalho (OLIVEIRA, 2003). Sendo assim, a HRA pode ser objetivada como a busca por qualidade ou estado de quem se pode confiar (PALLEROSI et al., 2011). Em termos práticos, a confiabilidade humana é estimada com a probabilidade de que uma tarefa ou serviço seja realizado com sucesso dentro de um tempo predefinido (LAFRAIA, 2001). Iida (1991) agrega que a HRA pode ser descrita como a probabilidade de uma tarefa ser desempenhada com sucesso pelo homem.

Na prática, a HRA pode ser definida como a estimativa da probabilidade de um sistema, em cujo propósito se busca estabelecer ações com sucesso de evitar erros humanos dentro de um período requerido (NASCIMENTO NETO, 2014).

\subsection{Análise dos modos de falhas e seus efeitos}

De acordo com a Associação Brasileira de Normas Técnicas (ABNT), na norma NBR 5462 (1994), a sigla FMEA (Failure Mode and Effects Analysis) é traduzida como Análise dos Modos de Falhas e seus Efeitos. A norma classifica esse método como um instrumento qualitativo de abordagem e análise da confiabilidade, com o intuito de estudar os modos de falhas verificados em um produto e, a partir disso, propor melhorias de funcionamento a esse produto, por meio do ranqueamento de prioridades. Pode-se dizer que o método busca reduzir as chances de ocorrência de falhas nos processos e, consequentemente, no produto final (LUZ et al., 2016).

Já Toledo e Amaral (2006) definiram FMEA como um método que busca avaliar e minimizar os riscos por meio de análise de possíveis falhas. Os objetivos da FMEA envolvem reconhecer e avaliar as falhas potenciais, identificar ações para eliminar ou reduzir a chance de ocorrência dessas falhas e, por fim, documentar o estudo para manter os padrões estabelecidos (FOGLIATTO e RIBEIRO, 2009).

Além disso, para Lafraia (2001) também são objetivos da FMEA identificar os testes necessários e os meios corretos para certificar um projeto e a criar um sistema lógico de operacionalidade com avaliações ou certificações de mudanças em projetos, processos ou materiais.

\subsection{Análise de árvores de falhas}

De acordo com Fogliatto e Ribeiro (2009), a FTA é um método sistemático para a analisar falhas e identificar suas causas. Os procedimentos de criação de uma árvore de 
falhas envolvem a seleção dos principais eventos a serem considerados como possíveis fontes de falhas no sistema em estudo (PALLEROSI, et al. 2011). A FTA pode ser uma análise do tipo qualitativa ou quantitativa. Assim, na análise qualitativa o foco é determinar as causas básicas de um evento ou a sequência que levou ao desencadeamento do mesmo (FOGLIATTO e RIBEIRO, 2009). Já na análise quantitativa, o objetivo é definir a probabilidade de ocorrência do evento (LAFRAIA, 2011).

Tanto qualitativa quanto quantitativa, a FTA permite mostrar o encadeamento de diferentes eventos associados a uma determinada falha ou evento de topo, em formato de árvore (LIMA et al., 2006). A FTA começa com o desenho que se distribuirá de forma descendente, que tem início com o seu evento de topo e segue para os demais eventos. Dessa forma, o desenho e o raciocínio são feitos do topo para baixo (FOGLIATTO e RIBEIRO, 2009). Assim, as falhas podem ser eventos associados com falhas de componentes, erro humano, falhas do sistema, assim como erros nos requisitos, erros de design e bugs em programas. Ou seja, quanto melhor for a definição do evento topo, mais fácil será a identificação das relações de dependência e possíveis causas de falhas.

\section{MÉTODO DE PESQUISA}

Inicialmente foi realizada uma pesquisa bibliográfica em livros, artigos e sites contendo informações sobre a proposta do tema, com o intuito de trazer esclarecimentos e fundamentar a pesquisa. Sendo assim, utilizou-se de acordo com os objetivos específicos do trabalho, uma pesquisa fundamentada em um estudo de revisão bibliográfica sobre o tema proposto para que haja o suprimento de conhecimento a todos que terão acesso a este trabalho.

De acordo com os objetivos, utilizou-se uma pesquisa exploratória, que para Gil (2008) é elaborada com o propósito de proporcionar uma visão geral, de caráter aproximativo, a respeito de determinado fato que ao final do processo, torna-se um problema compreensível, susceptível de investigação mediante uma sistematização de alguns procedimentos.

Quanto à classificação, pode se dizer que é também uma pesquisa aplicada, tendo como objetivo produzir conhecimento para a aplicação prática, voltado para solução específicas de problemas. A partir da aplicação do projeto em estudo se considera como 
uma pesquisa de classe quantitativa, pois permite tudo ser mensurável, onde traduz em números e categorias, para serem feitas as devidas análises (GIL, 2008).

Com relação à escolha da modalidade de pesquisa tem-se neste projeto um estudo de caso, que segundo Fachin (2006), se caracteriza por um estudo intensivo, onde se leva em conta a compreensão do assunto como um todo, do assunto investigado.

O objeto de estudo são as falhas humanas que ocorrem nos processos de atendimento a clientes e coleta de material biológico em um laboratório de análises clínicas, na cidade de Pelotas/RS. De posse dos dados de falhas, o propósito é identificar as causas e os riscos associados e condições de melhorias para evitá-las.

A coleta dos dados foi realizada com o auxílio de biomédicas que trabalham no laboratório, responsáveis pelo registro de não conformidades nos procedimentos de atendimento e coleta de material biológico. Obtiveram-se uma amostra de dados de três meses ininterruptos. Nesse período, registram-se 219 falhas humanas em um total de 13.258 atendimentos e coletas realizados.

Ao mesmo tempo em que se efetiva a coleta de dados, por observação direta e consulta a documentos faz-se o mapeamento dos dois processos sob análise, com subsequentes subdivisões em etapas.

Primeiramente, a análise consta de apurar os quantitativos dos dados de falhas e associá-los às etapas estabelecidas no mapeamento dos dois processos. As falhas são, a seguir, evidenciadas em erros e transgressões. Para isso, procede-se à análise qualitativa das falhas, com o auxílio dos colaboradores (biomédicas) do laboratório. Os erros são indicados como etapas dos processos realizadas de forma equivocada (má elaboração) relativamente aos padrões definidos. As transgressões são aquelas indicadas quando alguma das etapas dos processos é burlada (deixa de ser realizadas). Alguns poucos dados são excluídos da amostra de dados a ser analisada, a pedido da Gerência do Laboratório, para garantir o sigilo de clientes menores de idade, dependentes químicos e portadores de doenças sexualmente transmissíveis.

Em segundo lugar, identificam-se os riscos das falhas com aplicação da FMEA. Como terceiro procedimento, causas das falhas com maiores riscos são caracterizadas com o uso de FTA.

Por fim, interpretação com relação às alternativas para evitar a ocorrência de falhas são apresentadas. 


\section{ESTUDO DE CASO}

O laboratório de análises clínicas em estudo possui diversos padrões que devem ser cumpridos, a fim de proporcionar segurança e assertividade tanto para funcionários quanto para clientes. Foram observados diretamente e mapeados dois processos. O primeiro é o de atendimento ao cliente conforme a figura 1 a seguir:

Figura 1- Processo de Atendimento

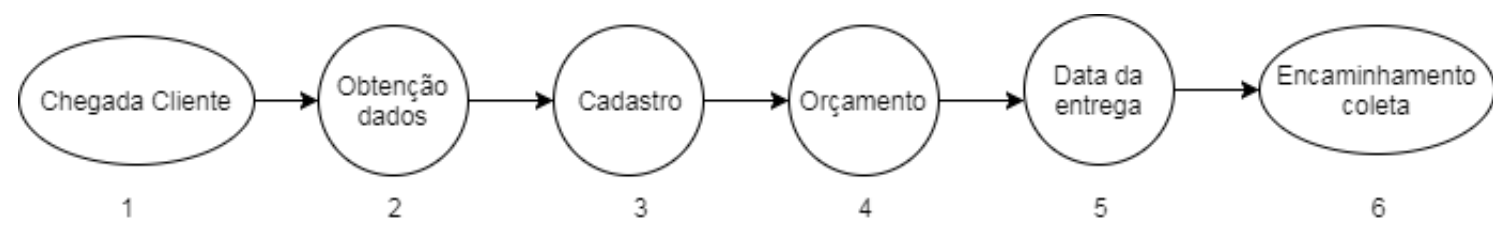

Fonte: Autores (2021)

O processo de atendimento engloba seis etapas. A primeira, é a chegada do cliente ao guichê de atendimento. Após isso, a atendente deve obter todos os dados relacionados ao cliente (nome, sexo, idade, endereço, etc) e ao seu médico, a fim de conferir os convênios de saúde contemplados. Depois disso, cadastram-se os dados do paciente no sistema, assim como os exames que o mesmo deseja fazer. Na quarta etapa, depois de verificar todos os exames a realizar e o convênio contemplado, deve-se repassar para o cliente o orçamento final do serviço. Com o pagamento realizado, a data de previsão de entrega dos resultados é definida. Por último, o cliente é encaminhado à sala de espera para aguardar o momento da coleta do material biológico.

O segundo processo, que dá continuidade ao atendimento ao cliente, é o de coleta de material biológico, detalhado na figura 2.

Figura 2 - Processo de coleta de material

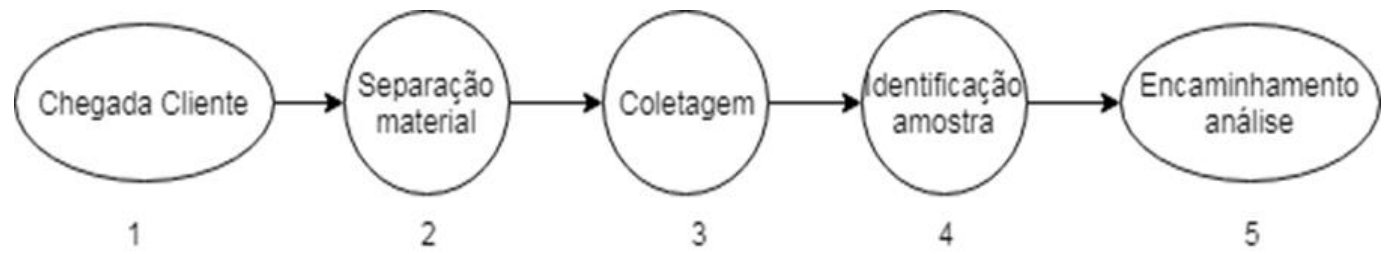

Fonte: Autores (2021)

O processo de coleta é constituído de cinco etapas principais. Na primeira, ocorre a chamada do cliente na recepção do laboratório e a chegada do mesmo na sala de coleta. 
Depois, a coletagem da amostra de material biológico é realizada. Posteriormente, todas as amostras são identificadas com duas etiquetas - uma gerada pelo sistema e outra manual, em que constam informações referentes ao nome, idade, horário de coletagem, tipo de exame (s) a ser realizado e ao médico que solicitou o exame. Por fim, os recipientes das amostras são encaminhados para os laboratórios de análises.

As 219 falhas encontradas nos processos de coleta e atendimento do laboratório foram dividas conforme o processo em que ocorreram, conforme a tabela 1.

Tabela 1 - Número de falhas nos processos de coleta e atendimento

\begin{tabular}{c|c}
\hline Processo & $\begin{array}{c}\text { Número de } \\
\text { Falhas }\end{array}$ \\
\hline Coleta & 62 \\
\hline Atendimento & 157 \\
\hline Total & 219 \\
\hline \multicolumn{2}{|c}{ Fonte: Autores (2021) }
\end{tabular}

Assim, é possível constatar que no processo de atendimento ocorrem mais falhas (72\%) em relação ao processo de coleta (28\%). Isso se deve, possivelmente, ao número de etapas do processo de atendimento, que é mais extenso e detalhista que o da coleta, pois contém informações repassadas pelo cliente, exigindo mais atenção dos funcionários para o preenchimento de formulário eletrônico e inclusão correta no sistema de informações do laboratório.

Na tabela 2 é possível ver o número de erros e transgressões presentes em cada processo.

Tabela 2 - Número de erros e transgressões dos processos de coleta e atendimento

\begin{tabular}{c|c|c}
\hline Processo & Erros & Transgressões \\
\hline Coleta & 8 & 54 \\
\hline Atendimento & 110 & 47 \\
\hline
\end{tabular}

Fonte: Autores (2021)

Verifica-se que no processo de coleta ocorrem mais transgressões do que no processo de atendimento. No processo de atendimento, as falhas, frequência e classificação entre erros e transgressões são apresentadas na tabela 3.

Tabela 3 - Tabela de falhas no processo de atendimento

\begin{tabular}{c|c|c|c}
\hline Processo & Falhas & Frequência & Classificação \\
\hline Atendimento & $\begin{array}{c}\text { Cadastro de exame } \\
\text { faltante }\end{array}$ & 16 & Transgressões \\
\hline
\end{tabular}




\begin{tabular}{|c|c|c|c|}
\hline Atendimento & $\begin{array}{l}\text { Cadastro de exame } \\
\text { incorreto }\end{array}$ & 41 & Erros \\
\hline Atendimento & $\begin{array}{l}\text { Data de nascimento } \\
\text { cliente incorreta }\end{array}$ & 1 & Erros \\
\hline Atendimento & $\begin{array}{l}\text { Data de promessa de } \\
\text { resultados incorreta }\end{array}$ & 35 & Erros \\
\hline Atendimento & $\begin{array}{c}\text { Exame não entregue } \\
\text { ao cliente }\end{array}$ & 3 & Transgressões \\
\hline Atendimento & $\begin{array}{l}\text { Exame sem entrada } \\
\text { no sistema }\end{array}$ & 28 & Transgressões \\
\hline Atendimento & $\begin{array}{l}\text { Nome do cliente } \\
\text { incorreto }\end{array}$ & 9 & Erros \\
\hline Atendimento & $\begin{array}{l}\text { Nome do médico } \\
\text { incorreto }\end{array}$ & 11 & Erros \\
\hline Atendimento & $\begin{array}{l}\text { Orçamento repassado } \\
\text { ao cliente incorreto }\end{array}$ & 10 & Erros \\
\hline Atendimento & $\begin{array}{l}\text { Sexo do cliente } \\
\text { incorreto }\end{array}$ & 3 & Erros \\
\hline
\end{tabular}

Fonte: Autores (2021)

Os erros são mais frequentes e suscetíveis de ocorrer no processo de atendimento, por se tratar de uma sequência de etapas que contém muitas informações a serem coletadas e incluídas no sistema de informações. A distribuição dessas falhas pode ser visualizada na figura 3.

Figura 3 - Frequência de falhas no atendimento

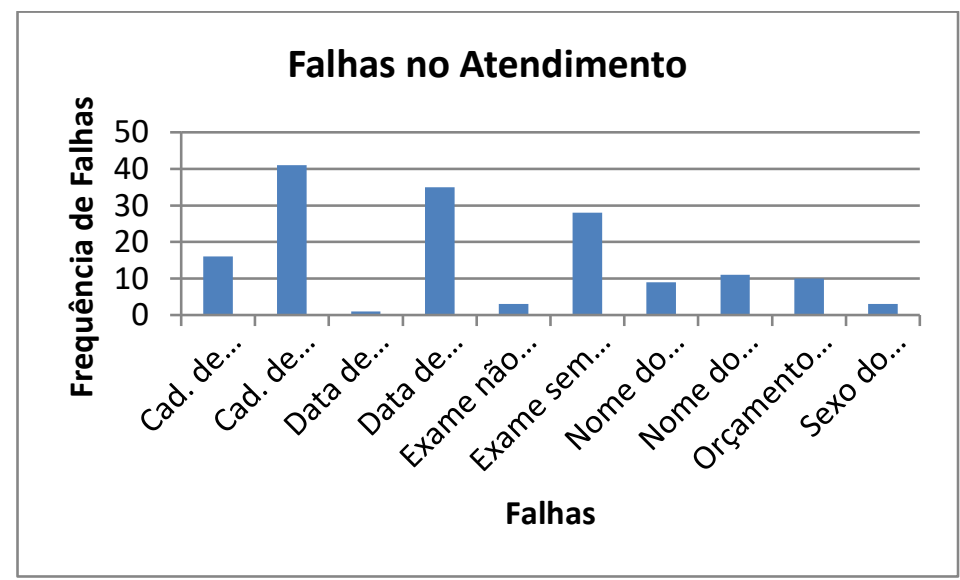

Fonte: Autores (2021)

A falha mais ocorrente no atendimento é a de cadastro de exame incorreto no sistema (26\%), contida na terceira etapa do processo (figura 1), e é fundamental para a correta análise das amostras coletadas. A segunda mais frequente é a data de entrega dos resultados repassada incorretamente para os clientes (22\%). Ela ocorre na quinta etapa do 
processo, é classificada como um erro, podendo gerar insatisfação e transtornos futuros para os clientes.

Na tabela 4, apresentam-se as falhas que ocorrem no processo de coleta.

Tabela 4 - Falhas no processo de coleta

\begin{tabular}{c|c|c|c}
\hline Processo & Falhas & Frequência & Classificação \\
\hline Coleta & $\begin{array}{c}\text { Identificação de } \\
\text { amostra de urina sem } \\
\text { etiqueta manual }\end{array}$ & 54 & Transgressões \\
\hline Coleta & $\begin{array}{c}\text { Mesma amostra com } \\
\text { horários diferentes }\end{array}$ & 8 & Erros \\
\hline
\end{tabular}

Fonte: Autores (2021)

As falhas identificadas no processo de coleta se dividem em dois tipos. A identificação de amostra de urina sem etiqueta manual corresponde a $87 \%$ do total e se repetiu 6,4 vezes mais em relação à falha de mesma amostra com horários diferentes. A frequência dessas falhas pode ser visualizada na figura 4.

Figura 4 - Frequência de falhas na coleta

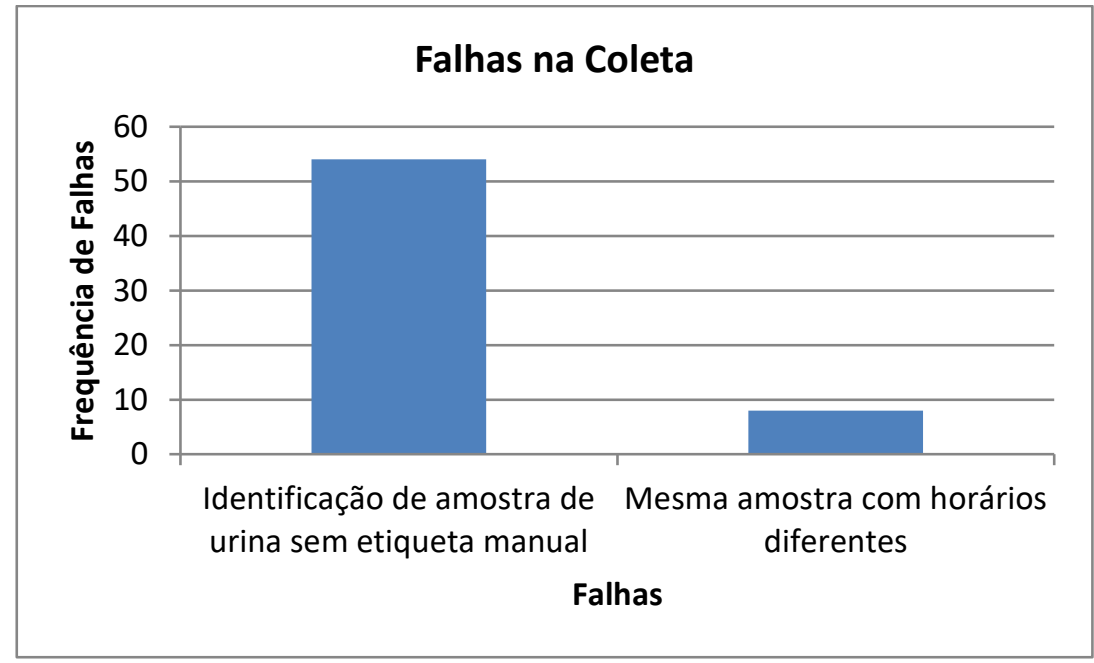

Fonte: Autores (2021)

A falha de identificação de amostra trata de uma transgressão que ocorre repetidas vezes na quarta etapa do processo de coleta. Ela ocorre, pois a amostra possui uma identificação gerada pelo sistema e a manual representa apenas a confirmação da amostra. Já a falha de horários diferentes ocorre na terceira etapa do processo e é classificada como um erro, sendo fundamental para a análise correta dos resultados.

Para cada processo estudado, foi aplicada a ferramenta FMEA, a fim de identificar o risco (RPN) de cada falha apurada anteriormente e propor o desenvolvimento correto 
das atividades. Dessa maneira, definiram-se os três parâmetros iniciais de avaliaçãodo do RPN: índice de severidade (S), ocorrência (O) e detecção (D).

O índice de severidade (S)varia de 10 a 1, em que 10 indica um efeito de gravidade elevado e relacionado à veracidade dos resultados e à confiabilidade do laboratório. A pontuação 1 indica nenhum efeito.

Em relação à detecção, o índice varia de 10 a 1, em que a pontuação 10 indica probabilidade de ocorrência de falhas igual ou maior que 100 a cada 1000 atendimentos. O índice 1 significa probabilidade remota de tais ocorrências.

O índice de detecção também varia de 10 a 1 , em que a pontuação 10 indica detecção quase improvável, pois o resultado não foi inspecionado e não existe forma de constar a falha. Já a pontuação 1 significa que a detecçao da falha vai ocorrer quase certamente. Ou seja, o controle é realizado em todos os casos em três vezes: visual, via sistema e diretamente com o cliente.

Assim o RPN é definido como a multiplicação de cada um dos índices, conforme a equação 1 .

$$
R P N=S x O x D
$$

Na figura 5 apresenta-se o risco (RPN) das falhas do processo de atendimento, assim como respectivos modos de falha, efeitos e causas potenciais, controle atual de prevenção e de detecção. 
Figura 5 - Tabela FMEA para o processo de atendimento

\begin{tabular}{|c|c|c|c|c|c|c|c|c|c|}
\hline & FMEA - Ar & lálise dos Mod & los de & Falhase Sel & Is Efe & itos & FME & $\overline{\mathrm{A}} \mathrm{N}$ № & \\
\hline $\begin{array}{l}\text { FMEA: Proces } \\
\text { PROCESSO: } \\
\text { RESPONSAV }\end{array}$ & $\begin{array}{l}\text { sso } \\
\text { Atendimento } \\
\text { EL: Supervisor }\end{array}$ & do Laboratório & $\begin{array}{l}\text { DATA } \\
\text { PREF }\end{array}$ & $\begin{array}{l}\text { A DE INICIO: } 0 \\
\text { ARADO POR: }\end{array}$ & $\begin{array}{l}1 / 02 / 2 \\
\text { Jorda }\end{array}$ & $\begin{array}{l}2018 \\
\text { ana }\end{array}$ & ISAO: 05/02/201 & & \\
\hline $\begin{array}{c}\text { FALHA DO } \\
\text { PROCESSO }\end{array}$ & $\begin{array}{c}\text { MODO DE } \\
\text { FALHA } \\
\text { POTENCIAL }\end{array}$ & $\begin{array}{c}\text { EFEITO (S) } \\
\text { DE FALHA } \\
\text { POTENCIAL }\end{array}$ & 㞻 & $\begin{array}{c}\text { CAUSA (S) } \\
\text { POTENCIAL } \\
\text { DE FALHA }\end{array}$ & 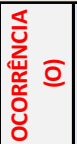 & $\begin{array}{c}\text { CONTROLE } \\
\text { ATUAL DE } \\
\text { PREVENÇÃO }\end{array}$ & $\begin{array}{l}\text { CONTROLE } \\
\text { ATUAL DE } \\
\text { DETECÇÃO }\end{array}$ & 皆 & 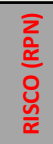 \\
\hline $\begin{array}{c}\text { Cadastro de } \\
\text { exame faltando }\end{array}$ & $\begin{array}{c}\text { Exame solicitado } \\
\text { pelo cliente não } \\
\text { foi colocado no } \\
\text { formulário }\end{array}$ & $\begin{array}{c}\text { Cliente não terá o } \\
\text { exame que } \\
\text { solicitou }\end{array}$ & 10 & $\begin{array}{l}\text { Desatenção dos } \\
\text { funcionários e } \\
\text { imprudência }\end{array}$ & 3 & \begin{tabular}{|c|} 
Reuniões \\
mensais sobre o \\
passo a passo do \\
atendimento
\end{tabular} & $\begin{array}{l}\text { Comparação das } \\
\text { requisições com o } \\
\text { cadastro }\end{array}$ & 3 & 90 \\
\hline $\begin{array}{c}\text { Cadastro de } \\
\text { exame incorreto }\end{array}$ & $\begin{array}{c}\text { Exame } \\
\text { cadastrado } \\
\text { divergente com o } \\
\text { solicitado }\end{array}$ & $\begin{array}{l}\text { Cliente terá um } \\
\text { exame diferente } \\
\text { do que solicitou }\end{array}$ & 10 & $\begin{array}{c}\text { Letra inlegível, } \\
\text { exames com } \\
\text { nomes } \\
\text { parecidos } \\
\end{array}$ & 5 & \begin{tabular}{|c|} 
Reuniões \\
mensais sobre o \\
passo a passo do \\
atendimento
\end{tabular} & $\begin{array}{l}\text { Comparação das } \\
\text { requisições com o } \\
\text { cadastro }\end{array}$ & 3 & 150 \\
\hline $\begin{array}{c}\text { Data de } \\
\text { nascimento do } \\
\text { cliente } \\
\text { incorreta } \\
\end{array}$ & \begin{tabular}{|c|} 
Data de \\
nascimento \\
cadastrada não é \\
a certa \\
\end{tabular} & $\begin{array}{l}\text { Divergência na } \\
\text { análise de } \\
\text { resultados }\end{array}$ & 10 & $\begin{array}{l}\text { Desatenção dos } \\
\text { funcionários }\end{array}$ & 1 & \begin{tabular}{|c|} 
Reuniões \\
mensais sobre o \\
passo a passo do \\
atendimento \\
\end{tabular} & $\begin{array}{c}\text { Conferência de } \\
\text { dados no momento } \\
\text { da coleta }\end{array}$ & 1 & 10 \\
\hline $\begin{array}{l}\text { Data de } \\
\text { promessa dos } \\
\text { resultados } \\
\text { incorreta }\end{array}$ & $\begin{array}{c}\text { Data de retirada } \\
\text { dos exames não é } \\
\text { a certa }\end{array}$ & $\begin{array}{c}\text { Cliente não terá } \\
\text { os exames na } \\
\text { data prevista }\end{array}$ & 9 & $\begin{array}{l}\text { Desatenção dos } \\
\text { funcionários, } \\
\text { erro de sistema }\end{array}$ & 5 & \begin{tabular}{|c|} 
Reuniões \\
mensais sobre o \\
passo a passo do \\
atendimento
\end{tabular} & $\begin{array}{c}\text { Comparação dos } \\
\text { exames com a } \\
\text { data prevista para } \\
\text { retirada }\end{array}$ & 6 & 270 \\
\hline $\begin{array}{l}\text { Exame não } \\
\text { entregue ao } \\
\text { cliente }\end{array}$ & $\begin{array}{c}\text { Exame não foi } \\
\text { disponibiliza do } \\
\text { para o cliente no } \\
\text { site }\end{array}$ & $\begin{array}{c}\text { Cliente não } \\
\text { conseguirá } \\
\text { visualizar seus } \\
\text { resultados }\end{array}$ & 9 & $\begin{array}{c}\text { Erro do sistema, } \\
\text { falta de } \\
\text { organização }\end{array}$ & 2 & \begin{tabular}{|c|} 
Reuniões \\
mensais sobre o \\
passo a passo do \\
atendimento \\
\end{tabular} & Não existe & 10 & 180 \\
\hline $\begin{array}{c}\text { Exame sem } \\
\text { entrada no } \\
\text { sistema }\end{array}$ & $\begin{array}{c}\text { Exame não foi } \\
\text { colocado na fila } \\
\text { de exames para } \\
\text { análise }\end{array}$ & $\begin{array}{c}\text { Exame não será } \\
\text { feito }\end{array}$ & 10 & $\begin{array}{c}\text { Erro do sistema, } \\
\text { falta de } \\
\text { organização }\end{array}$ & 4 & \begin{tabular}{|c|} 
Reuniões \\
mensais sobre o \\
passo a passo do \\
atendimento
\end{tabular} & $\begin{array}{l}\text { Comparação das } \\
\text { requisições } \\
\text { médicas com o } \\
\text { cadastro }\end{array}$ & 3 & 120 \\
\hline $\begin{array}{c}\text { Nome do cliente } \\
\text { incorreto }\end{array}$ & $\begin{array}{c}\text { Cadastro do nome } \\
\text { foi feito } \\
\text { erroneamente }\end{array}$ & $\begin{array}{l}\text { Nome do cliente } \\
\text { ficará incorreto } \\
\text { no sistema }\end{array}$ & 8 & $\begin{array}{c}\text { Desatenção dos } \\
\text { funcionários, } \\
\text { pressa }\end{array}$ & 3 & \begin{tabular}{|c|} 
Reuniões \\
mensais sobre o \\
passo a passo do \\
atendimento \\
\end{tabular} & $\begin{array}{c}\text { Conferência de } \\
\text { dados no momento } \\
\text { da coleta }\end{array}$ & 1 & 24 \\
\hline $\begin{array}{l}\text { Nome do } \\
\text { médico } \\
\text { incorreto }\end{array}$ & $\begin{array}{c}\text { Cadastro do nome } \\
\text { do médico foi } \\
\text { feito } \\
\text { erroneamente }\end{array}$ & $\begin{array}{l}\text { Nome do médico } \\
\text { ficará incorreto } \\
\text { no sistema }\end{array}$ & 6 & $\begin{array}{l}\text { Desatenção dos } \\
\text { funcionários, } \\
\text { pressa }\end{array}$ & 3 & \begin{tabular}{|c|} 
Reuniões \\
mensais sobre o \\
passo a passo do \\
atendimento
\end{tabular} & $\begin{array}{l}\text { Comparação das } \\
\text { requisições } \\
\text { médicas com o } \\
\text { cadastro }\end{array}$ & 3 & 54 \\
\hline $\begin{array}{l}\text { Orçamento } \\
\text { incorreto }\end{array}$ & $\begin{array}{c}\text { Orçamento } \\
\text { repassado ao } \\
\text { cliente não condiz } \\
\text { com o correto } \\
\end{array}$ & $\begin{array}{c}\text { Cobrança } \\
\text { indevida, } \\
\text { insatisfação do } \\
\text { cliente } \\
\end{array}$ & 7 & $\begin{array}{c}\text { Erro de } \\
\text { convênio, } \\
\text { desatenção dos } \\
\text { funcionários } \\
\end{array}$ & 3 & \begin{tabular}{|c|} 
Reuniões \\
mensais sobre o \\
passo a passo do \\
atendimento \\
\end{tabular} & Conferência de caixa & 2 & 42 \\
\hline $\begin{array}{l}\text { Sexo do cliente } \\
\text { incorreto }\end{array}$ & $\begin{array}{l}\text { Sexo do cliente } \\
\text { cadastrado não é } \\
\text { o correto }\end{array}$ & $\begin{array}{l}\text { Divergência na } \\
\text { análise de } \\
\text { resultados }\end{array}$ & 10 & $\begin{array}{c}\text { Desatenção dos } \\
\text { funcionários, } \\
\text { pressa }\end{array}$ & 2 & \begin{tabular}{|c|} 
Reuniões \\
mensais sobre o \\
passo a passo do \\
atendimento
\end{tabular} & $\begin{array}{l}\text { Conferência de } \\
\text { dados no } \\
\text { momento da } \\
\text { coleta } \\
\end{array}$ & 1 & 20 \\
\hline
\end{tabular}

Fonte: Autores (2021)

As falhas que apresentam maior risco são: data de promessa de resultados incorretas, exame não entregue ao cliente e cadastro de exames incorretos. Essas falhas estão ligadas diretamente a satisfação dos clientes e à confiabilidade do laboratório na prestação de serviços.

Na figura 6, mostra-se a tabela FMEA relacionada ao processo de coleta, assim como respectivos modos de falha, efeitos e causas potenciais, controle atual de prevenção e de detecção. 
Figura 6 - Tabela FMEA para o processo de coleta

\begin{tabular}{|c|c|c|c|c|c|c|c|c|c|}
\hline \multicolumn{7}{|c|}{ FMEA - Análise dos Modos de Falhas e Seus Efeitos } & \multicolumn{3}{|c|}{ FMEA № 2} \\
\hline \multicolumn{10}{|c|}{ FMEA: Processo } \\
\hline \multirow{2}{*}{\multicolumn{3}{|c|}{$\begin{array}{l}\text { PROCESSO: Coleta } \\
\text { RESPONSÁVEL: Supervisor do Laboratório }\end{array}$}} & \multicolumn{7}{|c|}{ DATA DE INÍCIO: 01/02/2018 } \\
\hline & & & PRE & PARADO POR & Jorda & ana & SÃO: 05/02/20 & & \\
\hline $\begin{array}{l}\text { FALHA DO } \\
\text { PROCESSO }\end{array}$ & $\begin{array}{c}\text { MODO DE } \\
\text { FALHA } \\
\text { POTENCIAL }\end{array}$ & $\begin{array}{c}\text { EFEITO (S) } \\
\text { DE FALHA } \\
\text { POTENCIAL }\end{array}$ & 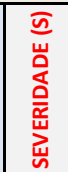 & $\begin{array}{c}\text { CAUSA (S) } \\
\text { POTENCIAL } \\
\text { DE FALHA }\end{array}$ & 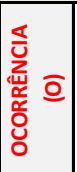 & $\begin{array}{c}\text { CONTROLE } \\
\text { ATUAL DE } \\
\text { PREVENÇÃO }\end{array}$ & $\begin{array}{l}\text { CONTROLE } \\
\text { ATUAL DE } \\
\text { DETECÇÃO }\end{array}$ & 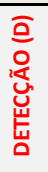 & 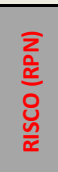 \\
\hline $\begin{array}{c}\text { Identificação de } \\
\text { amostra de } \\
\text { urina sem } \\
\text { etiqueta }\end{array}$ & $\begin{array}{c}\text { Segunda } \\
\text { identificação de } \\
\text { amostra não } \\
\text { realizada }\end{array}$ & $\begin{array}{c}\text { Falta da etiqueta } \\
\text { de confirmação } \\
\text { dos dados, } \\
\text { retrabalho }\end{array}$ & 9 & $\begin{array}{l}\text { Desatenção, } \\
\text { pressa }\end{array}$ & 6 & $\begin{array}{c}\text { Reuniões } \\
\text { mensais sobre o } \\
\text { passo a passo da } \\
\text { coleta }\end{array}$ & $\begin{array}{c}\text { Checagem pelas } \\
\text { biomédicas do } \\
\text { laboratório } \\
\text { visualmente }\end{array}$ & 6 & 324 \\
\hline $\begin{array}{c}\text { Mesma amostra } \\
\text { com horários } \\
\text { diferentes }\end{array}$ & $\begin{array}{c}\text { Amostras coletas } \\
\text { no mesmo horário } \\
\text { identificadas } \\
\text { erroneamente }\end{array}$ & $\begin{array}{l}\text { Divergência na } \\
\text { análise de } \\
\text { resultados }\end{array}$ & 10 & $\begin{array}{c}\text { Desatenção dos } \\
\text { funcionários, } \\
\text { pressa }\end{array}$ & 3 & $\begin{array}{c}\text { Reuniões } \\
\text { mensais sobre o } \\
\text { passo a passo da } \\
\text { coleta }\end{array}$ & $\begin{array}{c}\text { Checagem pelas } \\
\text { biomédicas do } \\
\text { laboratório } \\
\text { visualmente }\end{array}$ & 6 & 180 \\
\hline
\end{tabular}

Fonte: Autores (2021)

No processo de coleta, as duas falhas identificadas são bastante diferentes uma da outra e o risco que representam para o processo também. A primeira não-conformidade ocorre de forma mais recorrente que a segunda, por isso que o seu risco é mais elevado, apesar de a outra falha ter um grau de severidade maior.

Por meio do risco RPN estimados nas figuras 5 e 6 é possível caracterizar a prioridade de intervenção nas causas das falhas (tabela 5).

Tabela 5 - Tabela de prioridade de intervenção nas causas de falhas

\begin{tabular}{c|l}
\hline \multicolumn{1}{|c|}{ RPN } & \multicolumn{1}{c}{ Critério } \\
\hline 0 até 120 & $\begin{array}{l}\text { Menor: nenhuma ação será tomada (ou } \\
\text { tomada em longo prazo com a ótica de } \\
\text { melhoria contínua) }\end{array}$ \\
\hline 121 até 250 & $\begin{array}{l}\text { Moderado: ação a ser tomada em médio- } \\
\text { prazo. }\end{array}$ \\
\hline 521 até 1000 & $\begin{array}{l}\text { Alta: ação deve ser tomada e avaliação } \\
\text { detalhada deve ser feita em curto prazo. }\end{array}$ \\
\hline $\begin{array}{l}\text { Crítico: ação deve ser tomada de forma } \\
\text { imediata ou o processo interrompido } \\
\text { imediatamente. }\end{array}$ \\
\hline
\end{tabular}

Fonte: Autores (2021)

A fim de identificar as causas das falhas, é elaborado um diagrama de árvore para trangressões e erros do processo de atendimento para RPN superior a 120 (figuras 7 e 8). 
Figura 7 - Árvore de Falhas para as transgressões do processo de atendimento

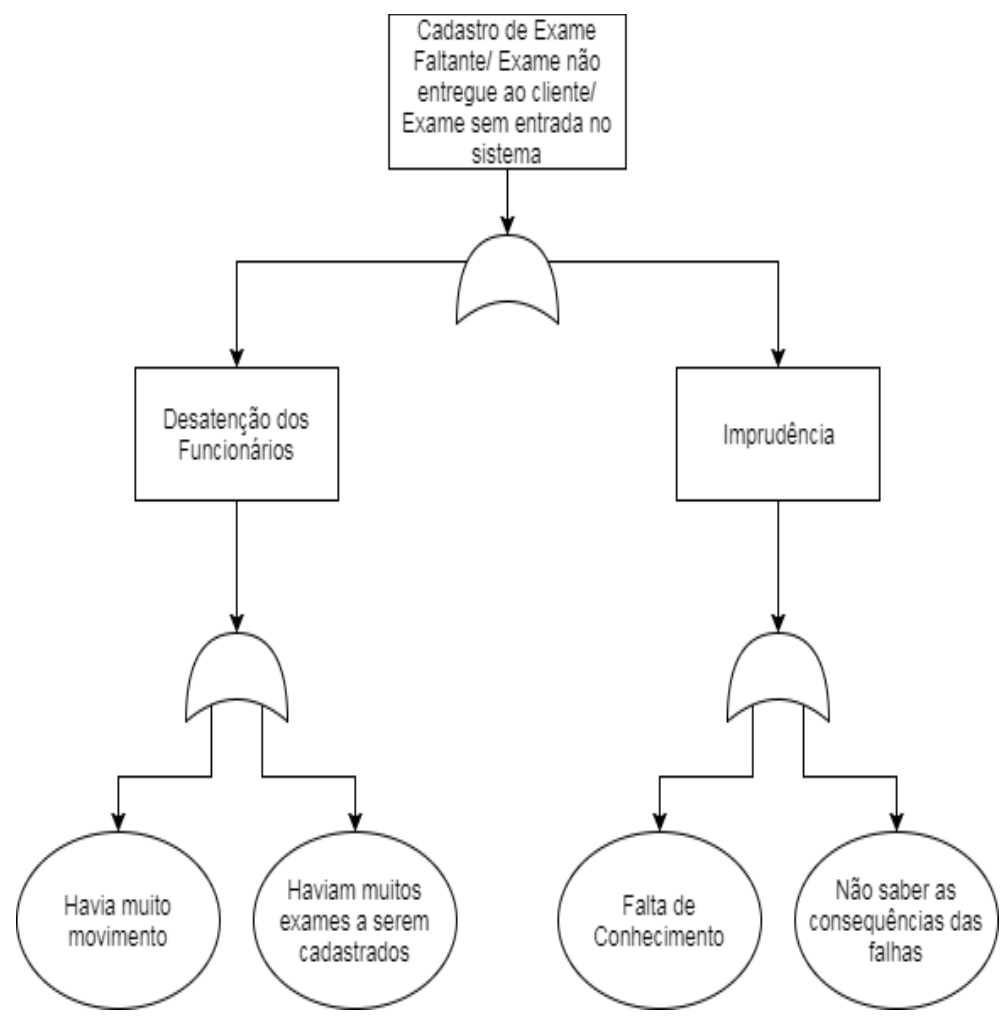

Fonte: Autores (2021)

Figura 8 - Árvore de Falhas para os erros do processo de atendimento

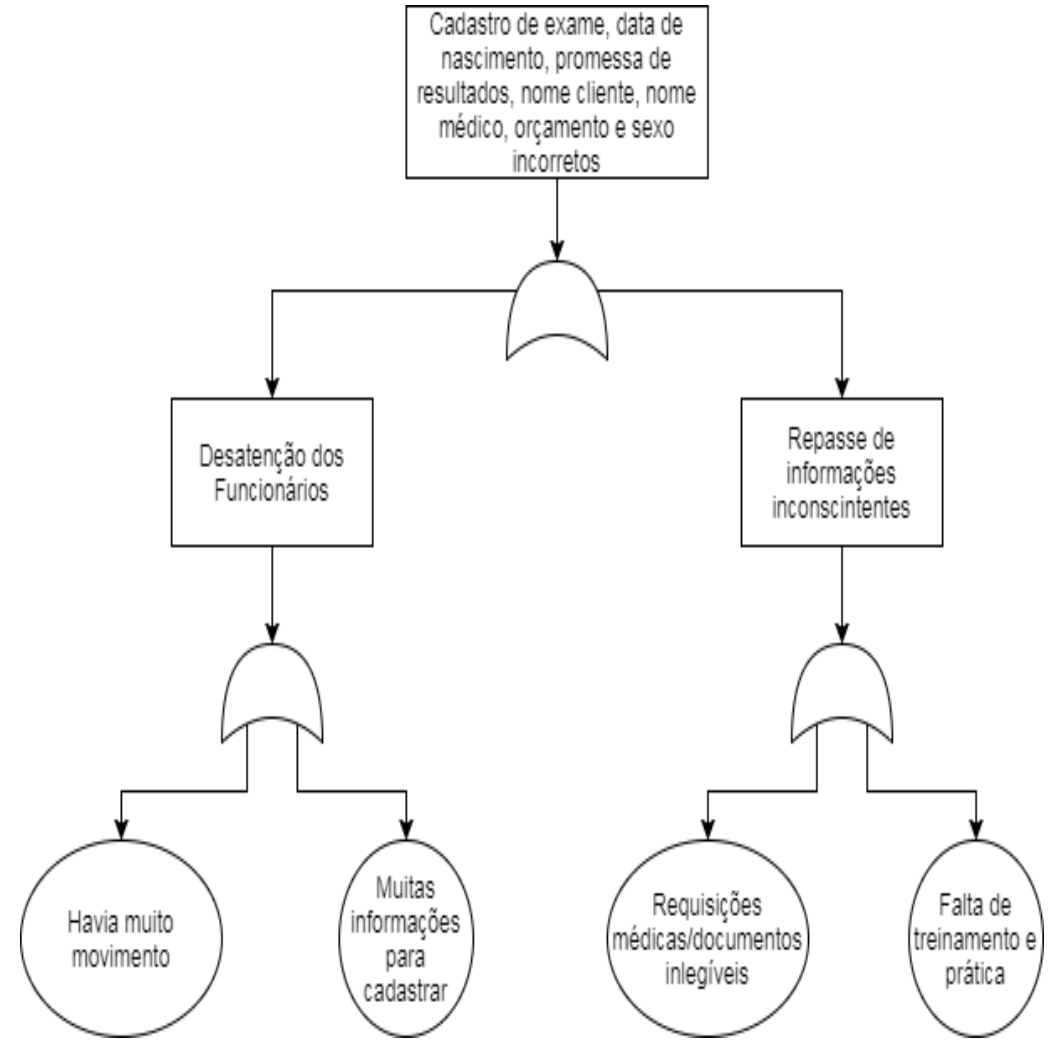

Fonte: Autores (2021) 
Em relação ao processo de coleta de material biológico, as árvores de falhas para a transgressão e o erro são mostradas nas figuras 9 e 10.

Figura 9 - Árvore de Falhas para as transgressões do processo de coleta

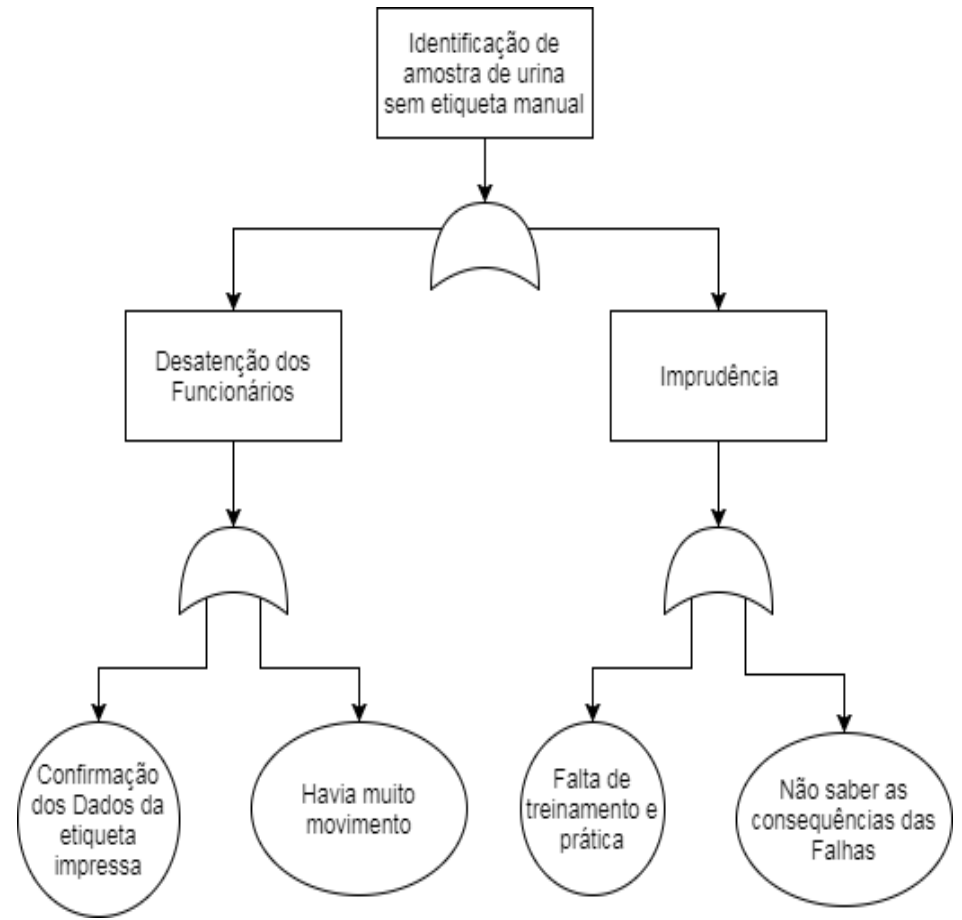

Fonte: Autores (2021)

Figura 10 - Árvore de Falhas para os erros do processo de coleta

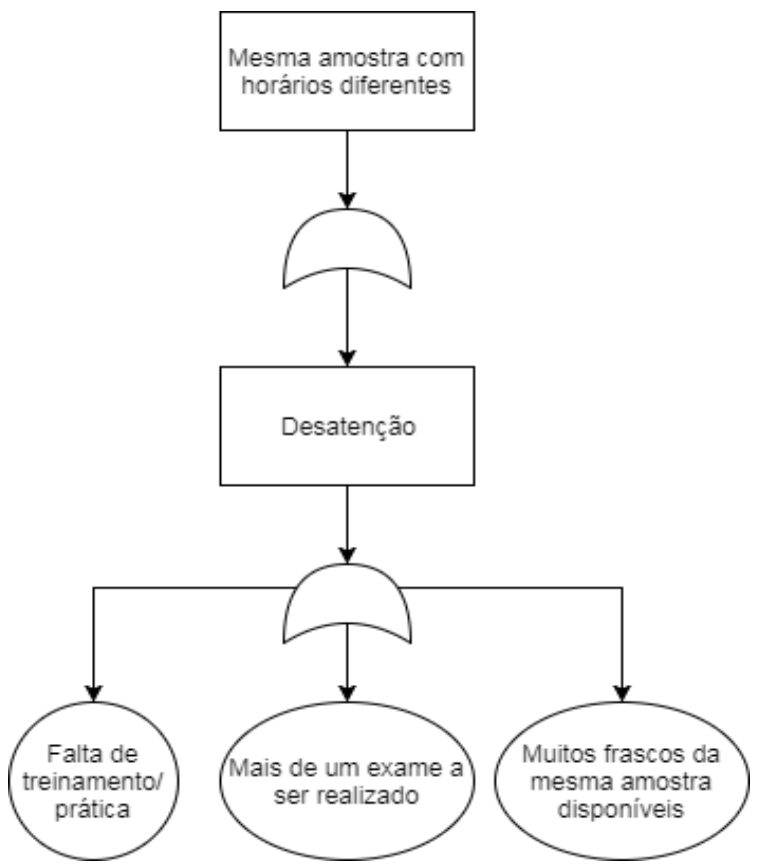

Fonte: Autores (2021) 


\section{DISCUSSÃO DOSRESULTADOS OBTIDOS}

Na classificação das não-conformidades, a representatividade de erros foi maior do que o total de transgressões e isso está ligado ao comportamento natural do homem. Constatou-se que as transgressões ocorridas são bastante direcionadas a tipos específicos de falhas - como burlar uma etapa do processo - e possuem menor variedade de ocorrência do que os erros.

A utilização das FMEA e FTA foi eficiente e subsidiou as investigações necessárias. Com a classificação das não-conformidades, percebe-se a diferença da origem das falhas e quais medidas podem ser adotadas a fim de minimizar o impacto e a recorrência das mesmas. Para isso, foi de extrema importância mapear os processos estudados, a fim de detectar quais etapas do atendimento e da coleta são cruciais para o bom desempenho dos mesmos.

Com a tabela FMEA, pôde-se descobrir o risco que cada falha representa para a sua execução. Foi possível perceber que no processo de atendimento ocorrem mais falhas que o processo de coleta e em sua maioria ligadas às transgressões. Com o risco final calculado, identificou-se no processo de coleta a falha com maior grau de prioridade de solução, visto que ela ocorre um grande número de vezes e gera retrabalho de confirmação de dados.

Já com a utilização do diagrama de árvores, foi possível observar de forma clara e objetiva a origem das não-conformidades apuradas e o que pode agravar o surgimento das mesmas. Para os erros, a desatenção, o ambiente movimentado, o grande número de etapas presentes nos processos contribuíram para o seu surgimento. Já as transgressões estão ligadas à falta de conhecimento técnico, à sensação de imprudência e ao não conhecimento das consequências do surgimento de falhas. Constata-se que o tratamento das causas das falhas pressupõe qualificação mais aprimorada das pessoas que atuam nos processos de atendimento e coleta de material bilógico no laboratório sob estudo.

Além disso, os procedimentos de predição, até então voltados para projetos e processos industriais, associados a falhas de equipamentos, puderam ser utilizados no controle e prevenção das falhas cometidas por pessoas. 


\section{CONTRIBUIÇÕES DA PESQUISA PARA A SOCIEDADE}

Neste estudo buscou-se aplicar de forma sistemática e integrada métodos qualitativos da confiabilidade como FMEA e FTA na análise de dados de confiabilidade humana em um estudo de caso realizado em um laboratório de análises clínicas. As falhas humanas foram classificadas em erros e transgressões. O Gap abordado e solucionado neste trabalho foi conciliar a aplicação sistemática e formalizada de métodos teóricos da confiabilidade para identificação e análise de falhas humanas em um problema real.

Desta forma, o método de pesquisa aplicado no estudo de caso contribui para sociedade, uma vez que a teoria e a prática são aplicadas no ambiente real de trabalho fomentando a cultura da análise de falhas dos processos e promovendo a divulgação de seus resultados.

\section{CONSIDERAÇÕES FINAIS}

Verifica-se que poucas publicações tratam da análise da confiabilidade humana. A aplicação de métodos qualitativos de confiabilidade para a análise da confiabilidade humana em processos reais estudados no laboratório de anaálises clínicas foi o objetivo alcançado deste trabalho.

Foram pontuados neste estudo os conceitos dos principais assuntos teóricos como métodos, ferramentas de confiabilidade. A metodologia de pesquisa do trabalho foi exploratória, aplicada com realização de estudo de caso único e específico. Foi realizada a aplicação dos métodos FMEA e FTA no estudo de caso. O estudo de caso foi realizado com sucesso quanto aos seus objetivos de identificação de falhas humanas em dois processos estudados atendimento e coletada de material.

Considera-se que, embora a metodologia proposta neste trabalho possa ser aplicada em outros processos similares do setor de serviços em estudo, o estudo de caso realizado é único com foco em uma empresa e, portanto, os resultados obtidos delimitamse ao escopo deste trabalho específico.

Os procedimentos de análise da confiabilidade, até então voltados para projetos e processos industriais, associados a falhas de equipamentos, puderam ser utilizados no controle e prevenção das falhas cometidas por pessoas. Conclui-se que os objetivos da pesquisa foram cumpridos e a pesquisa realizada vem a contribuir para o estudo da 
confiabilidade humana, cujo tema se mostra extremamente necessário de ser realizado no Brasil.

\section{REFERÊNCIAS}

Associação Brasileira de Normas Técnicas. NBR 5462 Confiabilidade e mantenabilidade. Rio de Janeiro, 1994.

Associação Brasileira de Normas Técnicas. NBR ISO 9000 Sistemas de gestão da qualidade, Fundamentos e vocabulário. Rio de Janeiro, 2000.

ALVARENGA, T. H. P. Mapeamento de processos: uma revisão. Ponta Grossa, PR: $\mathbf{8}^{\mathbf{o}}$ Encontro de Engenharia e Tecnologia dos Campos Gerais, 2013.

CARPPINETTI, L. C. R. Gestão da qualidade: conceitos e técnicas. 3. ed., Atlas, São Paulo, 2016.

COUTINHO, R. S.; GOMES, T. A. Facilitando a Melhoria de Processos de Negócio através da utilização de ferramentas de Workflow. Rio de Janeiro, 2012.

FACHIN, O. Fundamentos de Metodologia. $5^{\text {a }}$ ed. São Paulo: Editora Saraiva, 2006.

FERNANDES, J. M. R. (2005). Proposição de Abordagem Integrada de Métodos da Qualidade Baseada no FMEA. Curitiba: Pontifícia Universidade Católica do Paraná.

FOGLIATTO, F. S.; RIBEIRO J. L.D. Confiabilidade e Manutenção industrial. Elsevier, Rio de Janeiro, 2009.

IIDA, I. Novas abordagens em segurança do trabalho. Produção, Janeiro, v. 1, n. 2, 1991.

LAFRAIA, J. R. B. Manual de Confiabilidade, Mantenabilidade e Disponibilidade. Qualitymark, Rio de Janeiro, 2001.

LIMA, P. F. A., FRANZ, L. A. S, AMARAL, F. G. Proposta de utilização do FTA como ferramenta de apoio ao FMEA em uma empresa do ramo automotivo. XIII SIMPEP, Bauru, SP, 2006.

NASCIMENTO NETO, M. P. Proposição de uma sistemática para avaliação de Confiabilidade Humana em uma mina a céu aberto. Porto Alegre, RS, 2014.

OLIVEIRA, J. C. Segurança e saúde no trabalho: uma questão mal compreendida. São Paulo em Perspectiva, 17 (2), 3-12, 2013.

PALlEROSI, C. A., MAZZOLINI, B. P. M., MAZZOLINI, L. R. Confiabilidade Humana: conceitos, análises, avaliação e desafios. All Print, São Paulo, 2011.

SILVA, S. E. L. Falha Humana em Operação e Manutenção de Subestações. SENDI, Belo Horizonte, 2006.

SLACK, N., BRANDON-JONES, A., JOHNSTON, R. Administração da Produção. $4^{a}$ Ed., Atlas, São Paulo, 2015 
TOLEDO, J. C.; AMARAL, D. C. FMEA- Análise do Tipo e Efeito de Falha. UFSCar, São Carlos, 2006.

\section{Recebido em: 01/11/2021}

Aprovado em: 23/11/2021

Publicado em: 03/12/2021

\section{AUTORES:}

\section{Ariane Ferreira Porto Rosa}

Doutora em Automatique et Informatique Appliquées (Spécialité Génie Industriel Qualité), pela Université de Nantes.

Instituição: Universidade Federal de Pelotas, Centro de Engenharias, Curso de Engenharia de Produção.

Endereço: Rua Benjamin Constant, 989, Bairro Porto, Pelotas-RS, Brasil.

E-mail: afprosa61@gmail.com

\section{Jordana Rubin Brito}

Bacharel em Engenharia de Produção UFPel

Instituição: Centro de Engenharias, Universidade Federal de Pelotas

Endereço: Centro de Engenharias, Prédio da Antiga Cotada, Rua Benjamin Constant, 989 -

Porto, Pelotas - RS, Brasil - 96010-020

E-mail: jorubinb@gmail.com

\section{Rogério Royer}

Doutor em Administração de Empresas pela Universidade Federal do Rio Grande do Sul.

Instituição: Universidade Federal de Pelotas, Centro de Engenharias, Curso de Engenharia de Produção.

Endereço: Rua Benjamin Constant, 989, Bairro Porto, Pelotas-RS, Brasil.

E-mail: rogroyer@gmail.com

\section{Gilberto Tavares dos Santos}

Doutor em Engenharia de Produção pela Universidade Federal do Rio Grande do Sul. Instituição: Universidade Federal do Rio Grande do Sul, Escola de Administração. Endereço: Rua Washington Luiz, 855, Centro Histórico, Porto Alegre-RS, Brasil. E-mail: gilbertotav@ hotmail.com 